UW Biostatistics Working Paper Series

6-13-2003

\title{
Sensitivity Analysis for the Assessment of Causal Vaccine Effects on Viral Load in HIV Vaccine Trials
}

Peter B. Gilbert

Fred Hutchinson Cancer Research Center \& University of Washington, pgilbert@scharp.org

Ronald J. Bosch

Harvard University, rbosch@hsph.harvard.edu

Michael G. Hudgens

Fred Hutchinson Cancer Research Center ఓ University of Washington, mhudgens@scharp.org

\section{Suggested Citation}

Gilbert, Peter B.; Bosch, Ronald J.; and Hudgens, Michael G., "Sensitivity Analysis for the Assessment of Causal Vaccine Effects on Viral Load in HIV Vaccine Trials" (June 2003). UW Biostatistics Working Paper Series. Working Paper 208.

http://biostats.bepress.com/uwbiostat/paper208

This working paper is hosted by The Berkeley Electronic Press (bepress) and may not be commercially reproduced without the permission of the copyright holder.

Copyright (c) 2011 by the authors 


\section{Introduction}

Consider a randomized, two-arm, placebo-controlled clinical trial to evaluate efficacy of a preventive HIV vaccine. The first two trials of this kind began in 1998 and 1999, and are ongoing (Francis et al., 1998). For each trial, the primary objective is to assess the vaccine's impact on the incidence of HIV infection (Rida and Lawrence, 1995). Another objective of these trials and future trials is to assess the vaccine's impact on viral load post acquisition of HIV (Nabel, 2001); viral load is the concentration of HIV in blood or another body compartment. This objective is important because natural history studies have shown that the viral load of an infected person predicts infectiousness (Quinn et al., 2000) and the rate of disease progression (cf., Mellors et al., 1997), and several animal studies have identified vaccines that failed to prevent infection but successfully controlled viremia and prevented disease (cf., Shiver et al., 2002). Therefore, a vaccine effect to lower viral load may be beneficial, whereas an effect to increase viral load may hasten or exacerbate disease. The risk of harmful vaccine "enhancement" of viral load is genuine (Burke, 1992), and has been observed for several viral vaccines (cf., Mascola et al., 1992).

The impact of vaccination on viral load can be studied in several ways. The data available for analysis are right-censored HIV infection diagnosis times in all randomized subjects, and longitudinal quantitative measurements of viral load in subjects who become infected. Two main inferential approaches are intent-to-treat (ITT) analyses of all randomized subjects and conditional analyses of infected subjects only. The ITT approach assesses the causal effect of randomizing to vaccine. However, the majority (likely $>80 \%$ ) of randomized subjects will have zero viral load because they do not become infected during the trial, which can give ITT analyses low power for detecting many alternatives of interest (Hudgens, Hoering, and Self, 2002a). Also, in ITT analyses two very different populations (uninfected and infected subjects) are placed on the same response scale. 
Consequently, the ITT analysis of viral load assumes that the outcomes absence of infection (with zero viral load) and infection with viral load below the quantification limit of the assay are approximately equally prognostic for disease progression. This assumption is difficult to justify, because the initial suppression of viral load in infected subjects may be lost due to HIV evolution (Barouch et al., 2002). Alternatively, rank-based ITT methods could be used that assign the lowest two ranks to absence of infection and viral load below the assay limit, respectively. However, to achieve greater power and to study the causal effect of randomizing to vaccine in a subpopulation of persons who would become infected described below, in this article we consider conditional analyses.

Conditioning on infection poses a major challenge to making an unbiased inference of the vaccine effect on viral load, because the analyzed groups are selected by the postrandomization event HIV infection. This post-treatment selection bias problem is common in biomedical studies (cf., Rosenbaum, 1984; Robins and Greenland, 1992), and implies that a comparison of viral load between infected subgroups, which measures the 'net vaccine effect', does not have a causal interpretation. In particular, partial efficacy of the vaccine to prevent HIV infection can bias the viral load comparison. For example, the vaccine may prevent infections in individuals with strong immune systems, but allow infections in individuals with relatively weak immune systems. If a weaker immune system correlates with a higher viral load upon infection, then the viral loads in infected subjects will tend to be selectively shifted upwards in vaccine relative to placebo recipients. On the other hand, selection bias could occur in the opposite direction, for example the vaccine could protect well against highly virulent strains but allow infections with mild viruses which establish low viremia levels. Therefore, a standard two-sample test comparing viral loads between infected groups may give a misleading impression that vaccination enhances or suppresses viral burden. Or, the test may fail to detect a meaningful vaccine effect. 
Frangakis and Rubin (2002) (FR) developed a framework for causal inference that can be used for studying a causal effect of vaccine on viral load that adjusts for the postrandomization selection bias. This framework defines causal estimands using potential outcomes (Rubin, 1974, 1978; Holland, 1986). For the present problem, each trial participant has a potential infection status under each randomization assignment. Additionally, subjects who would be infected under randomization to vaccine have a potential viral load under vaccine assignment, and subjects who would be infected under randomization to placebo have a potential viral load under placebo assignment. Within FR's framework, a causal vaccine effect on viral load is defined as a comparison of potential viral loads under the two randomization assignments for a subgroup of subjects with a common pair of potential infection status outcomes; FR referred to such a group as a principal stratum.

Hudgens, Hoering, and Self (2002b) (HHS) developed tests for a causal vaccine effect on viral load in the "always infected" principal stratum of subjects who would be infected regardless of randomization to vaccine or placebo. Under plausible assumptions described in Section 2, vaccine recipients who become HIV infected would also be infected had they received placebo. Consequently, inferences drawn for the always infected subpopulation address a practical question for individuals vaccinated in a public health program: If I acquire HIV despite vaccination, what is the viral load compared to if I had foregone vaccination? We consider inference on causal estimands defined for the always infected principal stratum, which are defined in terms of potential outcomes in Section 2.

The causal estimands are not identified, because membership of an infected placebo recipient in the always infected principal stratum is unknown (i.e., the infection status had the subject been randomized to vaccine is unknown). This problem can be addressed by modeling the probability that an infected placebo recipient is in the always infected stratum as a function of the potential viral load under randomization to placebo. HHS 
implicitly took this approach, by defining two selection models that express bounds for the maximum plausible levels of selection bias. Under these models, which identify the estimands, HHS developed testing procedures for assessing differences in the potential viral load distributions of always infected subjects under the two randomization assignments.

Testing the null hypothesis presuming an extreme degree of selection bias is practically very useful, because rejection implies a significant effect of vaccination above and beyond any plausible selective effects. However, the actual degree of bias is likely less than that specified by an extreme model, so that HHS's tests may sacrifice power. Achieving maximal power is especially important for key subgroup analyses, such as by gender (Sterling et al., 2001), route of exposure, or host genotype, and for analyses of seminal viral load, given the higher variability of seminal versus plasma viral load (Coombs et al., 1998). Therefore, it is important to also consider selection models that reflect intermediate degrees of selection bias, which may be more realistic and will allow for more powerful statistical tests. In this article we develop a method for sensitivity analysis that considers a continuous range of possible selective effects spanning from no bias to maximal plausible bias as considered by HHS. In different contexts, Rosenbaum and Rubin (1983), Scharfstein, Rotnitzky, and Robins (1999), and Goetghebeur et al. (2000) also developed methods of continuously-indexed sensitivity analysis of inferences on causal effects.

The article is organized as follows. Section 2 defines causal estimands and shows that they are identified from three assumptions and a biased sampling model that specifies the nature and degree of selection bias. A class of logistic biased sampling models is described, which is indexed by an interpretable sensitivity parameter $\beta$ that can be chosen to represent any magnitude of selection bias ranging between extreme positive and negative bias. Given a particular model in the class, Section 3 describes procedures for testing the corresponding null hypothesis of no causal effect of vaccination on viral load. A plot 
of the test statistic (or $p$-value) versus $\beta$ provides a sensitivity analysis to help discern if the data support a causal effect that is robust to plausible post-randomization selective effects. Section 3 also shows how the sensitivity analysis can be based on estimation rather than testing. Section 4 evaluates the proposed testing procedures in simulations, and Section 5 illustrates a sensitivity analysis on a simulated vaccine trial dataset.

\section{Causal Estimands and Biased Sampling Models for Sensitivity Analysis}

\subsection{Definition and identifiability of causal estimands}

First we define the potential outcomes of the trial participants. Let $\mathbf{Z}$ be the vector of vaccination assignments for the $N$ randomized subjects, with $i^{\text {th }}$ element $Z_{i}\left(Z_{i}=v\right.$, vaccine; $Z_{i}=p$, placebo). Let $\mathbf{S}(\mathbf{Z})$ be the $N$-vector with $i$ th element $S_{i}(\mathbf{Z})$, which is

the indicator of whether the $i^{\text {th }}$ subject would be infected given $\mathbf{Z}$. For subjects with $S_{i}(\mathbf{Z})=1$, let $Y_{i}(\mathbf{Z}, \mathbf{S})$ be the potential viral load (PVL) given $\mathbf{Z}$ and $\mathbf{S}=\mathbf{S}(\mathbf{Z})$. In order to limit the possible potential outcomes for each subject, we adopt Rubin's (1978) Stable Unit Treatment Value Assumption (SUTVA) throughout. It states that $S_{i}(\mathbf{Z})=S_{i}\left(\mathbf{Z}^{\prime}\right)$ whenever $Z_{i}=Z_{i}^{\prime}$, and, $Y_{i}(\mathbf{Z}, \mathbf{S})=Y_{i}\left(\mathbf{Z}^{\prime}, \mathbf{S}^{\prime}\right)$ whenever $Z_{i}=Z_{i}^{\prime}$ and $S_{i}\left(Z_{i}\right)=S_{i}^{\prime}\left(Z_{i}\right)=1$. SUTVA implies that potential outcomes for each subject $i$ are unrelated to the assignment $Z_{j}$ of other subjects, and allows $S_{i}(\mathbf{Z})$ and $Y_{i}(\mathbf{Z}, \mathbf{S})$ to be written as $S_{i}\left(Z_{i}\right)$ and $Y_{i}\left(Z_{i}\right)$, respectively. Therefore, under SUTVA each subject has two potential infection outcomes $\left(S_{i}(v), S_{i}(p)\right)$ and at most two PVL outcomes $\left(Y_{i}(v), Y_{i}(p)\right)$. For each subject only one of $S_{i}(v)$ or $S_{i}(p)$ is observed, denoted $S_{i}^{o b s} \equiv S_{i}\left(Z_{i}\right)$, and in the subgroup with $S_{i}^{o b s}=1$, $Y_{i}^{o b s} \equiv Y_{i}\left(Z_{i}\right)$ is observed. Note that $Y_{i}(v)\left(Y_{i}(p)\right)$ is defined only if $S_{i}(v)=1\left(S_{i}(p)=1\right)$.

By Property 2 of FR, a comparison between the ordered sets $\left\{Y_{i}(v): S_{i}(v)=S_{i}(p)=\right.$ $1\}$ and $\left\{Y_{i}(p): S_{i}(v)=S_{i}(p)=1\right\}$ is a causal effect, because it is made within a principal stratum. For subjects in the always infected stratum $\left\{S_{i}(v)=S_{i}(p)=1\right\}$, suppose the $Y_{i}(v)$ are identically distributed as $F_{(v)}^{\text {alw.inf }}(\cdot)$, and the $Y_{i}(p)$ are identically distributed as 
$F_{(p)}^{a l w i n f}(\cdot)$. Then, any functional that measures a contrast of the distributions

$$
\begin{aligned}
& F_{(v)}^{\text {alw.inf }}(y) \equiv \operatorname{Pr}\left(Y_{i}(v) \leq y \mid S_{i}(v)=S_{i}(p)=1\right) \\
& F_{(p)}^{a l w . i n f}(y) \equiv \operatorname{Pr}\left(Y_{i}(p) \leq y \mid S_{i}(v)=S_{i}(p)=1\right)
\end{aligned}
$$

is a causal estimand. Based on (1), a null hypothesis for no causal effect of vaccination on viral load in the always infected principal stratum can be expressed as:

$$
H_{0}: F_{(v)}^{a l w . i n f}(y)=F_{(p)}^{a l w . i n f}(y) \text { for all } y .
$$

Unfortunately, because neither distribution in (1) is identifiable (since $S_{i}(v)$ and $S_{i}(p)$ are not both observed for any subject), it is not possible to test (2) without introducing assumptions. Two assumptions are useful for identifying the distributions:

A1: The assignment $Z_{i}$ of each subject is independent of his/her potential outcomes. A2: For each subject $i, \operatorname{Pr}\left(S_{i}(v)=1, S_{i}(p)=0\right)=0$.

Assumption A1 plausibly holds in HIV vaccine efficacy trials due to randomization and blinding. A2 states that no subject would be infected if randomized to vaccine but uninfected if randomized to placebo, and under A1 will hold if vaccination does not increase the per-exposure infection probability for any subject. The SUTVA assumption may not hold because HIV disease is infectious (Halloran and Struchiner, 1995); however if the study population is a small sample from a large population of susceptible individuals and there are few infectious contacts between trial participants, then it should approximately hold. SUTVA can be checked through epidemiologic studies and data on mixing of risk behavior among trial participants. Given SUTVA, A1 can be tested based on risk behavior data, and under SUTVA and A1, A2 can be checked by testing if the HIV infection rate is higher in the vaccine group than in the placebo group.

Assumption A2 is very useful, because it implies that infected vaccine recipients must be in the always infected principal stratum. Together with A1, this implies that 
$F_{(v)}^{a l w . i n f}(y)=F_{v}(y) \equiv \operatorname{Pr}\left(Y_{i}^{o b s} \leq y \mid S_{i}^{o b s}=1, Z_{i}=v\right)$, where $F_{v}(\cdot)$ is the distribution of viral load in infected vaccine recipients; thus $F_{(v)}^{a l w i n f}(\cdot)$ is identified from the observed data. A2 is similar to Angrist, Imbens, and Rubin's (1996) Monotonicity Assumption 5, which is useful for identifying a causal estimand defined for a principal stratum of compliers. On the other hand, A1 and A2 do not identify $F_{(p)}^{\text {alwinf }}(\cdot)$, because they do not determine whether an infected placebo recipient is in the "protected" $\left\{S_{i}(v)=0, S_{i}(p)=1\right\}$ or always infected $\left\{S_{i}(v)=1, S_{i}(p)=1\right\}$ stratum.

Given the randomization assignment and observed infection status of a trial participant, Table 1 indicates the principal stratum or strata to which the participant must belong, and lists the information available on potential viral loads. The table makes clear that the always infected stratum is the natural subpopulation for causal inference on viral load, because it is the only stratum for which causal estimands involve only well-defined potential viral loads. Rubin (2000) made this point through a parallel example in which there are two randomized treatments and vital status is observed one year after randomization, and the goal of causal inference is to assess the treatment effect on quality of life within the principal stratum of subjects alive under either treatment assignment.

\subsection{Logistic selection bias models that identify the causal estimands}

The set of subjects infected under randomization to placebo, $\left\{S_{i}(p)=1\right\}$, partitions into the principal strata of protected and always infected subjects, with the level of vaccine efficacy $(V E)$ against infection determining the proportion in each. Specifically, define $V E=1-R R=1-\operatorname{Pr}\left(S_{i}(v)=1\right) / \operatorname{Pr}\left(S_{i}(p)=1\right) ; V E$ is a causal estimand measuring the relative reduction in infection risk conferred by randomizing to vaccine versus placebo. A2 implies $V E=\operatorname{Pr}\left(S_{i}(v)=0 \mid S_{i}(p)=1\right)$, which is the probability that a subject in $\left\{S_{i}(p)=1\right\}$ is in the protected principal stratum (note that A2 is crucial here; $\operatorname{Pr}\left(S_{i}(v)=\right.$ $\left.0 \mid S_{i}(p)=1\right)$ is not identified by randomization alone). The density of $Y(p)$ in subjects 
infected under randomization to placebo $\left(f_{(p)}(y)\right)$ can be written as a mixture of the densities of $Y(p)$ for the protected $\left(f_{(p)}^{\text {prot }}(y)\right)$ and always infected $\left(f_{(p)}^{\text {alw.inf }}(y)\right)$ strata:

$$
f_{(p)}(y)=V E * f_{(p)}^{p r o t}(y)+(1-V E) * f_{(p)}^{\text {alw.inf }}(y)
$$

With some calculations, the mixture (3) can be re-expressed as a biased sampling model

$$
f_{(p)}^{\text {alw.inf }}(y)=W^{-1} w(y) f_{(p)}(y)
$$

where $w(y)=\operatorname{Pr}\left(S_{i}(v)=1 \mid Y_{i}(p)=y, S_{i}(p)=1\right)$ and $W=\int_{-\infty}^{\infty} w(y) f_{(p)}(y) d y$ is a normalizing constant equal to $1-V E=R R$. The weight function $w(y)=R R(y)=$ $1-V E(y)$ is the probability that a subject infected with viral load $y$ if randomized to placebo would be infected if randomized to vaccine.

Let $F_{p}(y)$ and $f_{p}(y)$ be the distribution and density of the observed viral load in infected placebo recipients, respectively. Under the randomization assumption A1, $F_{(p)}(y)=$ $F_{p}(y)$, and the biased sampling model (4) can be re-stated as $f_{(p)}^{\text {alw.inf }}(y)=(1-V E)^{-1} w(y)$ $f_{p}(y)$. Therefore, under A1-A2 the null hypothesis of interest (2) is equivalent to

$$
H_{0}: F_{v}(y)=(1-V E)^{-1} \int_{-\infty}^{y} w(z) d F_{p}(z) \text { for all } y
$$

By A1, $V E$ is identified from the observed data. If $w(\cdot)$ were known, then both $F_{(v)}^{a l w . i n f}(\cdot)$ and $F_{(p)}^{a l w . i n f}(\cdot)$ would be identified, and the hypothesis (2) could be tested. However, $w(\cdot)$ is unknown, and it is not possible to test whether a particular $w(\cdot)$ is correctly specified from the data plus A1-A2. Our approach to this problem assumes $w(\cdot)$ is known, and tests (5) for a variety of fixed choices of $w(\cdot)$. For such an approach to be fruitful, it is important that the unidentified sensitivity function $w(\cdot)$ be interpretable.

Towards this goal, we parameterize $w(y)$ as logistic, indexed by an interpretable selection bias parameter $\beta$, which allows it to be constant or smoothly monotone increasing or decreasing: $w(y)=w(y \mid \alpha, \beta)=\exp \{\alpha+\beta y\} /(1+\exp \{\alpha+\beta y\})$. The sensitivity parameter 
$\beta$ is a log odds ratio: $e^{\beta}$ is the odds ratio of infection under randomization to vaccine given infection under randomization to placebo with viral load $y$ versus with viral load $y-1$. This interpretation allows the choice of $\beta$ to be guided by beliefs about plausible degrees of selection bias. For fixed $\beta \in[-\infty, \infty]$, the logistic selection bias model is specified by

$$
F_{(p)}^{\text {alw.inf }}(y)=(1-V E)^{-1} \int_{-\infty}^{y} \frac{\exp \{\alpha+\beta z\}}{1+\exp \{\alpha+\beta z\}} d F_{p}(z) \equiv F_{p}(y \mid \beta)
$$

Given fixed $\beta$, the parameter $\alpha$ is determined as the solution to the equation $F_{p}(\infty \mid \beta)=1$.

Figure 1 illustrates five selection models specified by (6) and $\beta$ fixed at $-\infty,-1,0,1, \infty$, which represent different ways to distribute $V E$ of the mass of $f_{(p)}(y)$ into the protected principal stratum via (3). Note that if $V E=0$ there is no selection bias, regardless of $\beta$, and the higher $V E$, the greater opportunity for bias. Heuristically, $\beta$ specifies how much bias occurs through $V E$. Fixing $\beta=0$ specifies a constant weight $w(y \mid \alpha, \beta=0)=$ $R R$, and reflects an assumption of no selection bias. Fixing $\beta>0$ makes $w(y \mid \alpha, \beta)$ monotone increasing in $y$ and reflects "positive" selection bias, with infection odds under randomization to vaccine higher for a larger PVL $Y(p)=y$. In this case, if the causal null hypothesis $(2)$ is true, then the net vaccine effect is that $F_{v}(\cdot)$ is stochastically larger than $F_{p}(\cdot)$. Similarly, $\beta<0$ makes $w(y \mid \alpha, \beta)$ monotone decreasing in $y$ and reflects "negative" selection bias, with infection odds under randomization to vaccine lower for a larger $y$, and under $(2)$ the net vaccine effect is that $F_{v}(\cdot)$ is stochastically smaller than $F_{p}(\cdot)$.

HHS developed tests for (5), using two models representing maximum plausible positive and negative bias. HHS's "positive" selection model is specified by placing all subjects in $\left\{S_{i}(p)=1\right\}$ with $Y(p)$ less than the $V E^{\text {th }}$-percentile $q_{(p)}^{V E}$ of its distribution into the protected principal stratum, and the "negative" selection model is specified by placing all subjects in $\left\{S_{i}(p)=1\right\}$ with $Y(p)$ greater than the upper $V E^{\text {th }}$-percentile $q_{(p)}^{1-V E}$ of its distribution into the protected principal stratum. These models are limiting members of the class of logistic models (6), specified respectively by $\beta=\infty$ (Figure 1 , right-most 
panel) and $\beta=-\infty$ (Figure 1, left-most panel). To see this, note that setting $\alpha=-\beta q_{(p)}^{V E}$ implies $\lim _{\beta \rightarrow \infty} w(y \mid \alpha, \beta)$ equals $I\left\{y>q_{(p)}^{V E}\right\}$ for $y \neq q_{(p)}^{V E}$ and $1 / 2$ for $y=q_{(p)}^{V E}$, and setting $\alpha=-\beta q_{(p)}^{1-V E}$ implies $\lim _{\beta \rightarrow-\infty} w(y \mid \alpha, \beta)=I\left\{y<q_{(p)}^{1-V E}\right\}$ for $y \neq q_{(p)}^{1-V E}$ and $1 / 2$ for $y=q_{(p)}^{1-V E}$. Therefore, based on the logistic weight function with $\beta$ ranging between $-\infty$ to $\infty$, the class of models (6) spans all plausible magnitudes of selection bias.

If selection bias is presumed to follow model (6) for some unknown $\beta$ within a plausible range $\beta_{\text {neg }}$ to $\beta_{\text {pos }}$, then a 2 -sided null hypothesis representing no causal vaccine effect in the always infected stratum allowing for possible selection bias is given by

$$
H_{0 \beta_{p o s}, \beta n e g}: F_{p}\left(\cdot \mid \beta_{\text {pos }}\right) \leq F_{v}(\cdot) \leq F_{p}\left(\cdot \mid \beta_{\text {neg }}\right), \quad \beta_{\text {pos }} \in[0, \infty], \beta_{\text {neg }} \in[-\infty, 0] .
$$

Under A1-A2, $H_{0 \beta_{p o s}, \beta_{n e g}}$ is equivalent to $F_{(p)}^{\text {alw.inf }}(\cdot) \leq F_{(v)}^{\text {alw.inf }}(\cdot)$ assuming model (6) with $\beta=\beta_{\text {pos }}$ and $F_{(v)}^{\text {alw.inf }}(\cdot) \leq F_{(p)}^{\text {alw.inf }}(\cdot)$ assuming model (6) with $\beta=\beta_{\text {neg }}$. For the special case $\beta_{\text {pos }}=\beta_{\text {neg }}=0,(7)$ collapses to the null hypothesis of no net vaccine effect on viral load, $H_{0}: F_{v}(\cdot)=F_{p}(\cdot)$. Therefore, under the assumption of no selection bias, a standard comparison of viral load distributions between infected subgroups assesses the causal effect of vaccine in the always infected principal stratum.

One-sided null hypotheses representing no causal vaccine effect are given by

$$
\begin{array}{ll}
H_{0 \beta_{\text {pos }}}: F_{p}\left(\cdot \mid \beta_{\text {pos }}\right) \leq F_{v}(\cdot), & \beta_{\text {pos }} \in[0, \infty], \\
H_{0 \beta_{\text {neg }}}: F_{v}(\cdot) \leq F_{p}\left(\cdot \mid \beta_{\text {neg }}\right), & \beta_{\text {neg }} \in[-\infty, 0] .
\end{array}
$$

If (8) is rejected, then always infected individuals have significantly higher viral loads under randomization to vaccine than placebo when controlling for selection bias. In sum, A1-A2 and model (6) can be used to specify a 1- or 2-sided null hypothesis for no causal effect of vaccine in the always infected stratum that can be tested, and a sensitivity analysis can be performed by testing the hypothesis for a range of fixed values of $\beta_{\text {pos }}, \beta_{\text {neg }}$.

\section{Statistical Hypothesis Tests and Estimation}


Let $Y_{v 1}, \cdots, Y_{v n_{v}}$ and $Y_{p 1}, \cdots, Y_{p n_{p}}$ denote the samples of observed viral loads from infected vaccine and placebo recipients. $Y$ could be the average of 2 or more viral load measurements taken from an infected subject, or another continuous outcome such as the area under the longitudinal viral load curve. We assume each sample is independently, identically distributed, and the two samples are independent of one another. Sections 3.1 and 3.2 consider nonparametric tests of the null hypotheses (7), (8), and (9), and Section 3.3 considers nonparametric estimation of an average causal effect parameter.

\subsection{Nonparametric Test Statistics}

Fix $\beta_{\text {pos }} \geq 0$ and $\beta_{\text {neg }} \leq 0$. Using the empirical distributions $\widehat{F}_{v}$ and $\widehat{F}_{p}$ calculated from the two observed samples, and an estimate of $V E$, nonparametric tests of $H_{0 \beta_{\text {pos }}}, H_{0 \beta_{\text {neg }}}$, and $H_{0 \beta_{\text {pos }}, \beta_{\text {neg }}}$ can be based on comparisons of $\widehat{F}_{v}(\cdot)$ with $\widehat{F}_{p}\left(\cdot \mid \beta_{\text {pos }}\right), \widehat{F}_{p}\left(\cdot \mid \beta_{\text {neg }}\right)$, and both estimates, respectively. The $V E$ parameter can be estimated by $\widehat{V E}=1-\frac{n_{v}}{N_{v}} / \frac{n_{p}}{N_{p}}$, with $N_{v}\left(N_{p}\right)$ the number of subjects randomized to vaccine (placebo). Under A1 $\widehat{V E}$ is unbiased for $V E$ if the vaccine protects by an "all-or-none" mechanism, and is approximately unbiased if it protects by another mechanism, since HIV infection is a rare event (Halloran, Haber, and Longini, 1992). We consider three criterion functions for summarizing the comparisons, based on means, suprema, and integrated squared differences.

The statistic $T_{M \beta}$ for comparing means, appropriate for testing (8) or (9), is given by

$$
T_{M \beta}=\int_{-\infty}^{\infty} y\left\{d \widehat{F}_{v}(y)-d \widehat{F}_{p}(y \mid \beta)\right\}
$$

where $\int_{-\infty}^{\infty} y d \widehat{F}_{v}(y)=n_{v}^{-1} \sum_{i=1}^{n_{v}} Y_{v i}$ and $\widehat{F}_{p}(y \mid \beta)$ is the nonparametric maximum likelihood estimator of $F_{p}(y \mid \beta)$ under model (6), calculated as

$$
\widehat{F}_{p}(y \mid \beta)=(1-\widehat{V E})^{-1} \frac{1}{n_{p}} \sum_{i=1}^{n_{p}} I\left\{Y_{p i} \leq y\right\} w\left(Y_{p i} \mid \widehat{\alpha}, \beta\right) .
$$

Here, $\widehat{\alpha}$ is computed by solving the equation $\widehat{F}_{p}(\infty \mid \beta)=1$ for $\alpha$, i.e., $\alpha$ solves

$$
1-\widehat{V E}=\int_{-\infty}^{\infty} \frac{\exp \{\alpha+\beta y\}}{1+\exp \{\alpha+\beta y\}} d \widehat{F}_{p}(y)
$$


A solution to (11) can be found rapidly using a numerical one-dimensional line search.

The null hypothesis $H_{0 \beta_{\text {pos }}}$ is rejected if $T_{M \beta_{\text {pos }}}$ is large. For large positive $\beta_{\text {pos }}$ and $\widehat{\alpha}=-\beta_{\text {pos }} \widehat{q}_{p}^{\widehat{V E}}$, with $\widehat{q}_{p}^{\widehat{V E}}$ the $\left(n_{p}(1-\widehat{V E})\right)^{\text {th }}$ largest value of $Y_{p 1}, \cdots, Y_{p n_{p}}$ as used by HHS, $T_{M \beta_{\text {pos }}}$ reduces to HHS's nonparametric statistic $T_{M}$ that tests $(8)$ with $\beta_{\text {pos }}=\infty$. Similarly, $H_{0 \beta_{n e g}}$ is rejected if $T_{M \beta_{n e g}}$ is negative and large, and for large negative $\beta_{n e g}$ and $\widehat{\alpha}=-\beta_{n e g} \widehat{q}_{p}^{1-\widehat{V E}}, T_{M \beta_{n e g}}$ reduces to HHS's statistic $T_{M}$ that tests (9) with $\beta_{n e g}=-\infty$. The maximum of $\left|T_{M \beta_{p o s}}\right|$ and $\left|T_{M \beta_{n e g}}\right|$ can be used for a 2-sided test of (7).

Second, a 1-sided Kolmogorov-Smirnov-type statistic for testing (8) is defined by

$$
T_{K S \beta_{p o s}}=m^{1 / 2} \sup _{-\infty<y<\infty}\left|\left\{\widehat{F}_{p}\left(y \mid \beta_{\text {pos }}\right)-\widehat{F}_{v}(y)\right\} \vee 0\right|,
$$

with $n=n_{v}+n_{p}, m=\left(n_{v} n_{p}\right) / n$, and an Anderson-Darling-type statistic is defined by

$$
T_{A D \beta_{p o s}}=m \int_{-\infty}^{\infty} \frac{\left[\left\{\widehat{F}_{p}\left(y \mid \beta_{\text {pos }}\right)-\widehat{F}_{v}(y)\right\} \vee 0\right]^{2}}{\widehat{H}_{n}\left(y \mid \beta_{\text {pos }}\right)\left(1-\widehat{H}_{n}\left(y \mid \beta_{\text {pos }}\right)\right)} d \widehat{H}_{n}\left(y \mid \beta_{\text {pos }}\right)
$$

where $\widehat{H}_{n}\left(y \mid \beta_{\text {pos }}\right)=\left(n_{p} / n\right) \widehat{F}_{p}\left(y \mid \beta_{\text {pos }}\right)+\left(n_{v} / n\right) \widehat{F}_{v}(y)$. One-sided statistics for testing (9) are given by (12) and (13) with $\beta_{\text {pos }}$ replaced by $\beta_{\text {neg }}$ and $\vee$ replaced by $\wedge$. Two-sided statistics for testing (7) can be defined similarly. When $\beta=\beta_{\text {pos }}=\beta_{\text {neg }}=0$, the 2-sided statistics reduce to the classical Kolmogorov-Smirnov and Anderson-Darling test statistics for comparing two distribution functions (D'Agostino and Stephens, 1986).

\subsection{Computing Critical Values for the Tests}

We use a modification of the 'Controls Only' bootstrap procedure developed by HHS for computing critical values for the test statistics. The modification is that once the bootstrap estimate of vaccine efficacy $\widehat{V E}^{\star}$ is computed as in HHS, a bootstrap estimate $\widehat{\alpha}^{\star}$ is computed as the solution to equation (11) with $\widehat{V E}$ replaced by $\widehat{V E}^{\star}$. Estimating $V E$ and $\alpha$ within each bootstrap iteration appropriately accounts for the uncertainty in the vaccine efficacy estimate. 
In general, the nonparametric bootstrap tends to approximate smooth distributions better than distributions with discontinuities. For $\beta$ finite, the use of a smooth logistic selection weight function in the test statistics suggests that the nonparametric bootstrap should perform well. For $|\beta|$ infinite (the extreme cases), the distributions $F_{p}\left(\cdot \mid \beta_{\text {pos }}\right)$ and $F_{p}\left(\cdot \mid \beta_{\text {neg }}\right)$ have discontinuities at the truncation point, which could abrogate bootstrap performance. The simulation study confirms that tests of $H_{0 \beta_{p o s}}$ with $\beta_{\text {pos }}=\infty$ have poorer size and power characteristics than tests of $H_{0 \beta_{p o s}}$ with $\beta_{\text {pos }}=1$.

\subsection{Nonparametric Estimation}

Under A1-A2 and a model (6) with $\beta$ fixed, $\widehat{\mu}_{A C E}(\beta) \equiv-T_{M \beta}$ is a consistent estimate of the average causal effect (ACE) parameter $\mu_{A C E}(\beta)=\int_{-\infty}^{\infty} y\left\{d F_{(p)}^{\text {alw.inf }}(y)-\right.$ $\left.d F_{(v)}^{a l w . i n f}(y)\right\}$. By bootstrap re-sampling from $\widehat{F}_{v}(y)$ and $\widehat{F}_{p}(y \mid \beta), 95 \%$ bootstrap percentile confidence intervals about $\mu_{A C E}(\beta)$ can be constructed. An estimation-based sensitivity analysis can be carried out by plotting point and interval estimates of $\mu_{A C E}(\beta)$ versus $\beta$.

\section{Simulation Study}

Through simulations of an HIV vaccine trial we evaluate the three 1-sided tests of the null hypothesis $H_{0 \beta_{\text {pos }}}$ in (8). Rejecting (8) implies that individuals infected under either assignment have significantly higher viral load if assigned vaccine than if assigned placebo when controlling for selection bias specified by $\beta_{\text {pos }}$ and model (6). We consider an intermediate-sized efficacy trial with 45 infections expected in the placebo group (Rida et al., 1997), and suppose the true $V E$ equals $30 \%$ or $50 \%$. The true amount of selection bias is determined by the parameter $\beta=\beta_{\text {pos }}$ in model (6), with $\beta=0,1$, or $\infty$. Thus, data are generated under three kinds of null models, which assume no selection bias, an intermediate amount of selection bias (supposing the infection odds under vaccine of a subject who would be infected under placebo increases $e^{1}=2.72$-fold per one unit higher PVL $Y(p)$ ), and maximal plausible positive selection bias. We assume two independent 
measures of viral load are available per person. The sample $Y_{p 1}, \cdots, Y_{p n_{p}}$ is generated from a normal distribution with mean 4.50 and variance 0.36 . These parameter values equal those used by HHS, selected based on a cohort of recently HIV infected persons. The sample $Y_{v 1}, \cdots, Y_{v n_{v}}$ is generated from $F_{p}(\cdot \mid \beta)$ in model $(6)$ with true $\beta=0,1$, or $\infty$. For each true $\beta$, three vaccine effects on viral load in the always infected are evaluated: mean shifts of $\Delta=0,1 / 3$, or $1 / 2 \log _{10}$ over and above any selection bias induced by the true $\beta$, i.e., the samples are drawn such that $F_{(p)}^{\text {alw.inf }}(y)=F_{p}(y \mid \beta)$ and $F_{(v)}^{\text {alw.inf }}(y)=F_{p}(y-\Delta \mid \beta)$.

For each of 500 datasets simulated under each parameter configuration, the three 1sided test statistics are calculated, with presumed selection bias levels $\beta=0,1$, or $\infty$. Critical values for the tests are determined using 500 bootstrap replications.

Using a nominal 5\% Type I error level, Table 2 shows estimated sizes and powers of the tests. The sizes are judged by the bolded rows, for which the correct amount of selection bias is presumed (true $\beta=$ presumed $\beta$ ). All tests have empirical size close to nominal, except when $V E=30 \%$ and $\beta=\infty$ the size is inflated to $8-12 \%$. The elevated size is caused by the simulated trials with estimated $V E$ less than zero; this occurred 18 times and of these the nonparametric mean-based test rejected the null hypothesis 16 times. If the 18 trials with $\widehat{V E}<0$ are discarded, then the rejection rate is $5.8 \%$. A similar pattern was seen for the other test statistics. When $\widehat{V E}<0$, the testing procedure operates under the assumption of no selection bias, and simply tests $H_{0}: F_{v}(\cdot)=F_{p}(\cdot)$. However, in fact $F_{v}(\cdot)<F_{p}(\cdot)$ due to positive selection bias (true $\beta=\infty$ ), which explains the inflated probability of rejection. Note that when $V E=50 \%$ the sizes are not elevated, because the estimated $V E$ is rarely negative. In summary, the tests generally have nominal size, except that when $V E$ is low and the sample size is moderate, underestimation of $V E$ can lead to an increased risk of false rejection. This phenomenon was also found by HHS.

When the correct selection bias model is assumed, the three tests have comparable 
power, with that of the Kolmogorov-Smirnov procedure slightly less. In addition, power diminishes as the true $\beta$ increases. Next we consider power when an incorrect amount of selection bias is presumed (Table 2 , unbolded rows). If zero bias is presumed $(\beta=0)$, but in truth there is moderate bias $(\beta=1)$, power is high, but at the expense of an inflated false rejection rate, at $15 \%$ when $V E=30 \%$ and $26 \%$ when $V E=50 \%$. If zero bias is presumed and there is actually extreme bias $(\beta=\infty)$, then power is extremely high and the sizes are extremely inflated. This illustrates the importance of accounting for the possibility of selection bias to avoid being misled. Next, suppose there is no selection bias, but one conservatively presumes $\beta=\infty$. Then power drops severely, e.g., to $10 \%$ for detecting a $1 / 2 \log _{10}$ mean shift when $V E=50 \%$, compared to $93 \%$ if the correct $\beta=0$ is assumed. For the more moderate assumption $\beta=1$, a much smaller price is paid, with power dropping to $64 \%$. Thus, making a highly conservative assumption of maximal selection bias can cause great power loss. This finding supports the use of a continuously-indexed sensitivity analysis as proposed here.

The Kolmogorov-Smirnov-type and Anderson-Darling-type tests are expected to have greater power than the mean-based test for detecting non-mean-shift alternatives. We briefly studied this conjecture by generating placebo group viral loads from a normal mixture distribution $0.5 N(3.50,0.36)+0.5 N(5.50,0.36)$ (e.g., infection with a mild or virulent virus), and vaccine group viral loads from a mixture of truncated normal distributions $0.5 \operatorname{TruncN}(3.50,0.36)+0.5 \operatorname{TruncN}(5.50,0.81)$, with truncation point of each distribution at the $70^{\text {th }}$ percentile. With alternative hypothesis no change in the first component and a $1.5 \log _{10}$ mean shift in the second component, assuming $\beta=\infty$, the Kolmogorov-Smirnov-type test had $78 \%$ power while the other tests had between $13 \%$ and $20 \%$ power. Thus, if the viral load distributions are expected to differ in respects other than a mean-shift, then the Kolmogorov-Smirnov-type test may be preferable. 


\section{Example}

To illustrate how a sensitivity analysis could be carried out on a forthcoming vaccine trial dataset, we analyze a single dataset, simulated using Gaussian distributions assuming $n_{p}=45$ infections in the placebo group, $\widehat{V E}=40 \%$ (and thus $n_{v}=27$ infections in the vaccine group), a true causal vaccine effect to reduce the mean viral load in the always infected by $0.33 \log _{10}$, and true $\beta=\beta_{\text {neg }}=-1$, i.e., moderate negative selection bias that leads to lower viral loads in infected vaccine recipients. The true causal and biasing vaccine effects on viral load imply that the net vaccine effect on mean viral load is 0.49 $\log _{10}$. For $\beta_{n e g}$ ranging in $[-\infty, 0]$, we consider testing $H_{0 \beta_{n e g}}$ in $(9)$ versus the alternative hypothesis that vaccination lowers viral load in the always infected.

The first step is to produce descriptive plots and summary measures comparing the observed viral load distributions between the infected subgroups. The average viral loads are 3.96 and 4.48 in the infected vaccine and placebo groups, respectively. The second step is to calculate a test statistic for values of $\beta=\beta_{\text {neg }}$ ranging between 0 and a negative value that makes the selection bias odds ratio $O R=e^{-\beta}$ large (e.g., $\beta=-5$ yields $e^{-\beta}=148$ ), and for the extreme model $(\beta=-\infty)$. The third step is to plot the $p$-value of the test statistic versus $O R$, which will always be monotone except for stochastic variations in the bootstrap. This provides a graphical sensitivity analysis (Figure 2). Fourth, calculation of the value of $\beta$ at which the test statistic is exactly statistically significant at the 0.025 level allows one to assess the extent of selection bias needed to lose the significance of the result. A 0.025 significance level is chosen because the test is 1-sided. In this example the critical $\beta$ value for the $T_{M \beta}$ test statistic is -1.83 , which implies the selection odds ratio must be at least $e^{1.83}=6.23$ before the significance of the test result is lost. The sensitivity analyses based on the other two test statistics give similar results (Figure 2).

Fifth, an estimation-based sensitivity analysis can be carried out (Figure 3). Suppose 
vaccination must lower mean viral load in the always infected by at least $0.2 \log _{10}$ to be considered clinically significant. The value of $\beta$ at which the lower $95 \%$ confidence limit for $\mu_{A C E}(\beta)$ crosses 0.2 is -0.50 , corresponding to a "critical" odds ratio of $e^{0.50}=1.65$.

Sixth, the analyses could be repeated for important subgroups of infected participants. Seventh, interpretations are made. In this example, a study team might conclude that it is unlikely that selection bias could fully explain the observed lower viral loads in infected vaccine recipients, and therefore a genuine viral suppressing effect of vaccine in the always infected is inferred. However, whether the effect is clinically significant is inconclusive. These conclusions would be based on beliefs that a selection bias effect with odds ratio 6.23 or higher is implausible, but a selection odds ratio of 1.65 is not unexpected.

Note that if only the hypothesis $H_{0 \beta_{n e g}}$ with extreme selection bias $\beta_{n e g}=-\infty$ had been tested, then the team would likely not be able to conclude that vaccination reduced viral load in the always infected ( $p$-value $>0.20$, Figure 2$)$. This illustrates the added value of a continuously-indexed sensitivity analysis.

\section{Discussion}

Appropriate interpretation of analyses of vaccine effects on viral load is challenging. Two main reasons are the lack of validation of viral load measures as accurate surrogates for secondary transmission and disease progression, and the potential for selective effects of the vaccine to bias inferences. Like HHS, we address the second problem, and extend their work to provide a method of sensitivity analysis over a continuous range of levels of putative selective effects. Since the true amount of selection bias may be considerably less than the worst-case amounts considered by HHS, the methods developed here may provide for more powerful assessments.

As illustrated in the Example, an observation of lower viral loads in infected vaccine recipients compared to infected placebo recipients could be caused partly by a causal 
viral suppressing effect of vaccine in the always infected principal stratum and partly by selective vaccine protection against viruses that produce higher viral loads. Both effects are beneficial, and the assessment of the net vaccine effect (in the infected subgroups) usefully informs about the overall benefit of vaccination, and this result should be reported together with the causal inference for the always infected principal stratum. On the other hand, for assessing a possible vaccine effect to increase viral load, the inference on the net vaccine effect could dangerously mislead. Selection bias could create higher viral loads in infected vaccine recipients compared to infected placebo recipients, i.e., produce a negative net vaccine effect, even though the vaccine has no adverse causal effect on viral load and has beneficial $V E>0$. Therefore, it is crucial to build robustness to selection bias into assessments of vaccine harm, to protect against a spurious conclusion that could prevent use of or slow development of a safe and partially efficacious vaccine.

Within the framework of FR, this article develops techniques for causal inference in the always infected principal stratum. Alternatively, causal inference could be made using a missing data framework that assumes all randomized subjects will eventually become HIV infected, and thus at some point will have a viral load value. In such an approach, the viral load is missing in subjects who have not yet been infected by the time of the analysis, and causal estimands can be defined based on functionals of contrasts of the viral load distributions for the vaccine and placebo groups. The goal of assessing such estimands is to compare the viral load distribution between the randomized groups had (contrary to fact) all subjects been infected during the trial. Rotnitzky and Robins (1997) developed an inverse probability of censoring weighted estimating equations method that could be used for causal inference on a mean-difference version of this estimand. This technique would model the viral load by a semiparametric conditional mean model with unspecified error distribution and the infection probability (i.e., the response probability) by a parametric 
model. If the hazard rate of infection rather than the binary infection probability was modeled, then Scharfstein, Rotnitzky, and Robins' (1999) method would apply for making inference on the same estimand. The advantages of these approaches include that they minimize modeling assumptions, they can incorporate predictors of the infection risk, and they can be used for sensitivity analysis of the effect of misspecification of the model for infection risk. The drawback of any such missing data approach for the present application is that the causal estimand may not be relevant or interpretable, because it is unrealistic to suppose that all subjects would eventually be HIV infected. FR criticize use of such a causal estimand because it uses nonexistent "a priori" counterfactuals. Inferences for the always infected subpopulation provide interpretable and practical information for vaccine recipients who become HIV infected despite vaccination.

In addition to HIV vaccine trials, the methods developed here apply to general randomized clinical trials, for sensitivity analyses of causal treatment effects in the subpopulation of subjects who would experience a post-randomization event under either assignment.

\section{ACKNOWLEDGEMENTS}

The authors thank Andrea Rotnitzky, Steve Self, the Associate Editor and referees for helpful comments. This work was supported by NIH grants AI46703-01 and AI38855.

\section{REFERENCES}

Angrist, J., Imbens, G.W., and Rubin, D.B. (1996). Identification of causal effects using instrumental variables (with discussion). Journal of the American Statistical Association 91, 444-472.

Barouch, D.H., Kunstman, J., Kuroda, M.J., et al. (2002). Eventual AIDS vaccine failure in a rhesus monkey by viral escape from cytotoxic $\mathrm{T}$ lymphocytes. Nature 415, 335-339. 
Burke, D.S. (1992). Human HIV vaccine trials: Does antibody-dependent enhancement pose a genuine risk? Perspectives in Biology and Medicine 35, 511-530.

Coombs, R.W., Speck, C.E., Hughes, J.P., et al. (1998). Association between culturable human immunodeficiency virus type 1 (HIV-1) in semen and HIV-1 RNA levels in semen and blood: evidence for compartmentalization of HIV-1 between semen and blood. Journal of Infectious Diseases 177, 320-330.

D’Agostino, R.B. and Stephens, M.A. (1986). Goodness-of-fit techniques. Marcel Dekker, New York.

Francis, D.P., Gregory, T., McElrath, M.J., et al. (1998). Advancing AIDSVAX to phase 3: safety, immunogenicity, and plans for phase 3. AIDS Research and Human Retroviruses 14 Suppl 3, S325-S331.

Frangakis, C.E. and Rubin D.B. (2002). Principal stratification in causal inference. Biometrics 58, 21-29.

Goetghebeur, E., Kenward, M., Molenberghs, G., and Vansteelandt, S. (2000). Inferential tools for sensitivity analysis and noncompliance in clinical trials. Proceedings of the Annual Meeting. Indianapolis, Indiana: American Statistical Association.

Halloran, M.E., Haber M.J., and Longini I.M. (1992). Interpretation and estimation of vaccine efficacy under heterogeneity. American Journal of Epidemiology 136, 328-343.

Halloran, M.E. and Struchiner, C.J. (1995). Causal inference in infectious diseases. Epidemiology 6, 142-151.

Holland, P. (1986). Statistics and causal inference. Journal of the American Statistical Association 81, 945-961.

Hudgens, M.G., Hoering, A., and Self, S.G. (2002a). On the analysis of viral load end- 
points in HIV vaccine trials. In: Abstracts of the Fourteenth International AIDS Conference 2002, Barcelona, Spain, July 2002.

Hudgens, M.G., Hoering, A., and Self, S.G. (2002b). On the analysis of viral load endpoints in HIV vaccine trials. Statistics in Medicine: in press

Mascola, J.R., Mathieson, B.J., Zack, P.M., Walker, M.C., Halstead, S.B., and Burke, D.S. (1993). Summary report: workshop on the potential risks of antibody-dependent enhancement in human HIV vaccine trials. AIDS Research and Human Retroviruses 9, 1175-1184.

Mellors, J.W., Munoz, A., Giorgi, J.V., et al. (1997). Plasma viral load and CD4+ lymphocytes as prognostic markers of HIV-1 infection. Annals of Internal Medicine 126, 946-954.

Nabel, G.J. (2001). Challenges and opportunities for development of an AIDS vaccine. Nature 410, 1002-1007.

Quinn, T.C., Wawer, M.J., Sewankambo, N., et al. (2000). Viral load and heterosexual transmission of human immunodeficiency virus type 1. New England Journal of Medicine 342, 921-929.

Rida, W., Fast, P., Hoff, R., and Fleming, T.R. (1997). Intermediate-sized trials for the evaluation of HIV vaccine candidates: a workshop summary. Journal of Acquired Immune Deficiency Syndrome 16, 195-203.

Rida, W.N. and Lawrence, D.L. (1995). Prophylactic HIV vaccine trials. In: Finkelstein DM, Schoenfeld DA, eds. AIDS clinical trials: Guidelines for design and analysis. Wiley-Liss, New York, 319-348.

Robins, J.M. and Greenland, S. (1992). Identifiability and exchangeability of direct and 
indirect effects. Epidemiology 3, 143-155.

Rosenbaum, P.R. (1984). The consequences of adjustment for a concomitant variable that has been affected by the treatment. The Journal of the Royal Statistical Society, Series A 147, 656-666.

Rosenbaum, P.R. and Rubin, D.B. (1983). Assessing sensitivity to an unobserved binary covariate in an observational study with binary outcome. The Journal of the Royal Statistical Society, Series B 45, 212-218.

Rotnitzky, A. and Robins, J. (1997). Analysis of semi-parametric regression models with non-ignorable non-response. Biometrics 16, 81-102.

Rubin, D.B. (1974). Estimating causal effects of treatments in randomized and nonrandomized studies. Journal of Educational Psychology 66, 688-701.

Rubin, D.B. (1978). Bayesian inference for causal effects. Annals of Statistics 6, 34-58.

Rubin, D.B. (2000). Comment on "Causal inference without counterfactuals," by A.P. Dawid. Journal of the American Statistical Association 95, 435-437.

Scharfstein, D.O., Rotnitzky, A., and Robins, J.M. (1999). Adjusting for nonignorable drop-out using semiparametric nonresponse models. Journal of the American Statistical Association 94, 1096-1146.

Shiver, J.W., Fu, T.-M., Chen, L., et al. (2002). Replication-incompetent adenoviral vaccine vector elicits effective anti-immunodeficiency virus immunity. Nature 415, $331-335$.

Sterling, T.R., Vlahov, D., Astemborski, J., Hoover, D.R., Margolick, J.B., and Quinn, T.C. (2001). Initial plasma HIV-1 RNA levels and progression to AIDS in women and men. New England Journal of Medicine 344, 720-725. 
Table 1. For the two randomization assignments $Z_{i}=v, p$ and infection outcomes $S_{i}^{o b s} \equiv S_{i}\left(Z_{i}\right)=0,1$, the table indicates the basic principal stratum or strata to which the subjects belong, and the information available on the potential viral loads $Y_{i}(v)$ and $Y_{i}(p)$. Note that $Y_{i}(Z)$ is defined if and only if $S_{i}(Z)=1, Z=v, p$, and the principal strata of uninfected placebo recipients and of infected vaccine recipients are known by assumption A2.

\begin{tabular}{|c|c|c|c|c|}
\hline $\begin{array}{c}\text { Randomiz. } \\
\text { Assignm. } \\
Z_{i}\end{array}$ & $\begin{array}{c}\text { Observed } \\
\text { Infection } \\
\text { Status } S_{i}^{o b s}\end{array}$ & \multicolumn{3}{|c|}{$\begin{array}{c}\text { Principal Stratum }\left\{S_{i}(v), S_{i}(p)\right\} \text { and } \\
\text { Information on Potential Viral Loads } Y_{i}(v), Y_{i}(p)\end{array}$} \\
\hline vaccine & uninfected & $\begin{array}{l}\text { Protected } \\
\left\{S_{i}(v)=0, S_{i}(p)=1\right\} \\
Y_{i}(v) \text { undefined } \\
Y_{i}(p) \text { unobserved }\end{array}$ & or & $\begin{array}{l}\text { Never-infected } \\
\left\{S_{i}(v)=0, S_{i}(p)=0\right\} \\
Y_{i}(v) \text { undefined } \\
Y_{i}(p) \text { undefined }\end{array}$ \\
\hline placebo & uninfected & $\begin{array}{l}\left\{S_{i}(v)=1, S_{i}(p)=0\right\} \\
(\text { empty set by A2) }\end{array}$ & & $\begin{array}{l}\text { Never-infected } \\
\left\{S_{i}(v)=0, S_{i}(p)=0\right\} \\
Y_{i}(v) \text { undefined } \\
Y_{i}(p) \text { undefined }\end{array}$ \\
\hline vaccine & infected & $\begin{array}{l}\left\{S_{i}(v)=1, S_{i}(p)=0\right\} \\
(\text { empty set by A2) }\end{array}$ & & $\begin{array}{l}\text { Always-infected } \\
\left\{S_{i}(v)=1, S_{i}(p)=1\right\} \\
Y_{i}(v) \text { observed } \\
Y_{i}(p) \text { unobserved }\end{array}$ \\
\hline placebo & infected & $\begin{array}{l}\text { Protected } \\
\left\{S_{i}(v)=0, S_{i}(p)=1\right\} \\
Y_{i}(v) \text { undefined } \\
Y_{i}(p) \text { observed }\end{array}$ & or & $\begin{array}{l}\text { Always-infected } \\
\left\{S_{i}(v)=1, S_{i}(p)=1\right\} \\
Y_{i}(v) \text { unobserved } \\
Y_{i}(p) \text { observed }\end{array}$ \\
\hline
\end{tabular}


Table 2 . Power $\times 100 \%$ for detecting a $0,1 / 3$, and $1 / 2 \log _{10}$ mean-shift alternative, over and above any selection bias induced by the true $\beta$, based on a 1-sided $5 \%$ level test

\begin{tabular}{|c|c|c|c|c|c|c|c|c|c|c|}
\hline \multirow{2}{*}{$\begin{array}{c}\text { True } \\
\beta \\
\end{array}$} & \multirow{2}{*}{$\begin{array}{c}\text { Presumed } \\
\beta\end{array}$} & \multicolumn{3}{|c|}{$\begin{array}{c}\text { Nonparametric } \\
\text { Mean }\end{array}$} & \multicolumn{3}{|c|}{$\begin{array}{l}\text { Kolmogorov- } \\
\text { Smirnov }\end{array}$} & \multicolumn{3}{|c|}{$\begin{array}{l}\text { Anderson- } \\
\text { Darling }\end{array}$} \\
\hline & & 0 & $1 / 3$ & $1 / 2$ & 0 & $1 / 3$ & $1 / 2$ & 0 & $1 / 3$ & $1 / 2$ \\
\hline 0 & 0 & 4.8 & 73.8 & 96.8 & $\begin{array}{c}V E= \\
5.4\end{array}$ & $\begin{array}{l}30 \% \\
\mathbf{6 9 . 0}\end{array}$ & 91.2 & 4.0 & 72.0 & 94.4 \\
\hline 0 & 1 & 3.2 & 49.8 & 82.0 & 3.0 & 44.8 & 78.2 & 2.4 & 46.8 & 79.6 \\
\hline 0 & $\infty$ & 0.2 & 15.8 & 36.0 & 0.8 & 16.8 & 37.6 & 0.2 & 15.4 & 33.6 \\
\hline 1 & 0 & 14.6 & 91.8 & 99.8 & 15.6 & 85.4 & 97.8 & 14.4 & 90.2 & 99.2 \\
\hline 1 & 1 & 5.8 & 67.6 & 94.6 & 7.2 & 63.4 & 90.8 & 5.4 & 65.4 & 94.0 \\
\hline 1 & $\infty$ & 1.8 & 26.0 & 52.2 & 1.4 & 27.6 & 51.8 & 1.6 & 25.8 & 48.8 \\
\hline$\infty$ & 0 & 69.6 & 100 & 100 & 76.2 & 100 & 100 & 79.8 & 100 & 100 \\
\hline$\infty$ & 1 & 37.4 & 95.8 & 100 & 42.6 & 97.0 & 100 & 45.8 & 97.4 & 100 \\
\hline$\infty$ & $\infty$ & 8.8 & 55.0 & 83.2 & 12.0 & 55.2 & 82.4 & 10.8 & 57.2 & 86.2 \\
\hline 0 & 0 & 6.6 & 70.0 & 93.2 & $\begin{array}{c}V E= \\
5.8\end{array}$ & $\begin{array}{l}50 \% \\
\mathbf{6 3 . 8}\end{array}$ & 89.8 & 5.8 & 66.4 & 92.6 \\
\hline 0 & 1 & 1.0 & 30.6 & 64.2 & 0.4 & 25.8 & 58.0 & 0.2 & 26.6 & 61.2 \\
\hline 0 & $\infty$ & 0.2 & 3.4 & 10.2 & 0.4 & 3.6 & 9.0 & 0.2 & 2.8 & 7.4 \\
\hline 1 & 0 & 25.8 & 92.2 & 99.0 & 24.8 & 87.4 & 99.0 & 24.0 & 91.0 & 99.2 \\
\hline 1 & 1 & 5.0 & 62.4 & 88.2 & 5.8 & 57.2 & 84.2 & 4.2 & 60.6 & 87.8 \\
\hline 1 & $\infty$ & 0.4 & 9.8 & 30.6 & 0.2 & 8.6 & 25.0 & 0.2 & 8.2 & 24.6 \\
\hline$\infty$ & 0 & 94.8 & 100 & 100 & 97.2 & 100 & 100 & 97.6 & 100 & 100 \\
\hline$\infty$ & 1 & 56.8 & 99.2 & 100 & 72.6 & 99.8 & 100 & 69.0 & 99.6 & 100 \\
\hline$\infty$ & $\infty$ & 5.2 & 59.2 & 84.2 & 6.0 & 55.6 & 79.4 & 6.4 & 57.8 & 82.0 \\
\hline
\end{tabular}


Figure Legends

Figure 1. The upper panel shows plots of the density of $Y_{i}(p)$ for subjects infected under randomization to placebo $\left\{S_{i}(p)=1\right\}$ (total area) partitioned into the subdensity for "protected" subjects not infected under randomization to vaccine $\left\{S_{i}(v)=0, S_{i}(p)=1\right\}$ (hatchmarked area $=V E$ ) and the subdensity for subjects "always infected" under randomization to either vaccine or placebo $\left\{S_{i}(v)=1, S_{i}(p)=1\right\}$ (unshaded area $=1-V E$ ). Using model (6) with $\beta=-\infty,-1,0,1$, or $\infty$, the 5 panels reflect different assumptions about how the vaccine relative risk $w(y \mid \alpha, \beta)=R R(y)=\operatorname{Pr}\left(S_{i}(v)=1 \mid Y_{i}(p)=y, S_{i}(p)=\right.$ 1) depends on the potential viral load (PVL) $Y_{i}(p)=y$ for subjects infected under randomization to placebo. The lower panel shows corresponding plots of the logistic weight function $w(y \mid \alpha, \beta)$. The hatchmarked areas equal $V E=0.30$, and $\alpha$ was calculated from $1-V E=\int_{-\infty}^{\infty} w(z \mid \alpha, \beta) d F_{(p)}(z)$ with $F_{(p)}(\cdot)$ given a normal distribution.

Figure 2. Based on the nonparametric mean-based, Anderson-Darling-type, and KolmogorovSmirnov-type test statistics, the figure shows the 1-sided bootstrap $p$-value plotted as a function of the selection bias odds ratio $O R=e^{-\beta}=e^{-\beta_{n e g}} ; e^{\beta}$ is the odds ratio of infection under randomization to vaccine given infection under randomization to placebo with viral load $y$ versus with viral load $y-1$. If the magnitude of selection bias is believed to be less than $O R=e^{1.83}=6.23$, then a significant causal effect of vaccination to lower viral load in the always infected principal stratum can be inferred.

Figure 3. Point estimates $\widehat{\mu}_{A C E}(\beta)=-T_{M \beta}$ (bold line) and bootstrap $95 \%$ confidence intervals (dotted lines) for the average causal effect of vaccine $\mu_{A C E}(\beta)=\int_{-\infty}^{\infty} y\left\{d F_{(p)}^{a l w . i n f}(y)-\right.$ $\left.d F_{(v)}^{\text {alw.inf }}(y)\right\}$ in the always infected principal stratum as a function of the selection bias odds ratio $O R=e^{-\beta_{\text {pos }}}$ (left side of 0$)$ and of $O R=e^{-\beta_{\text {neg }}}$ (right side of 0 ); $e^{\beta}$ is the odds 
ratio of infection under randomization to vaccine given infection under randomization to placebo with viral load $y$ versus with viral load $y-1$. If the magnitude of selection bias is believed to be less than $O R=e^{0.50}=1.65$, then a significant causal effect of vaccination to lower the mean viral load by at least $0.2 \log _{10}$ in the always infected principal stratum can be inferred. 

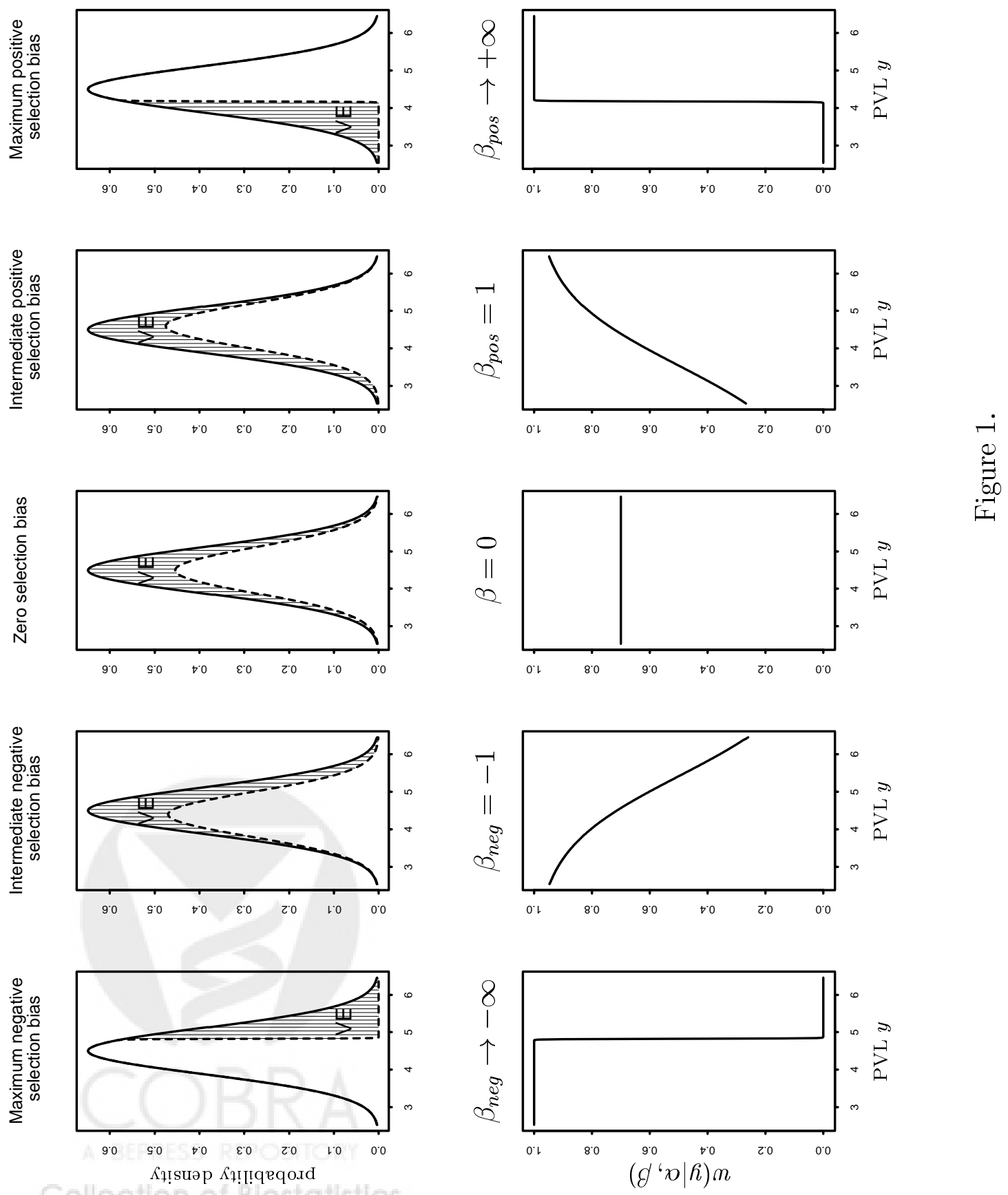


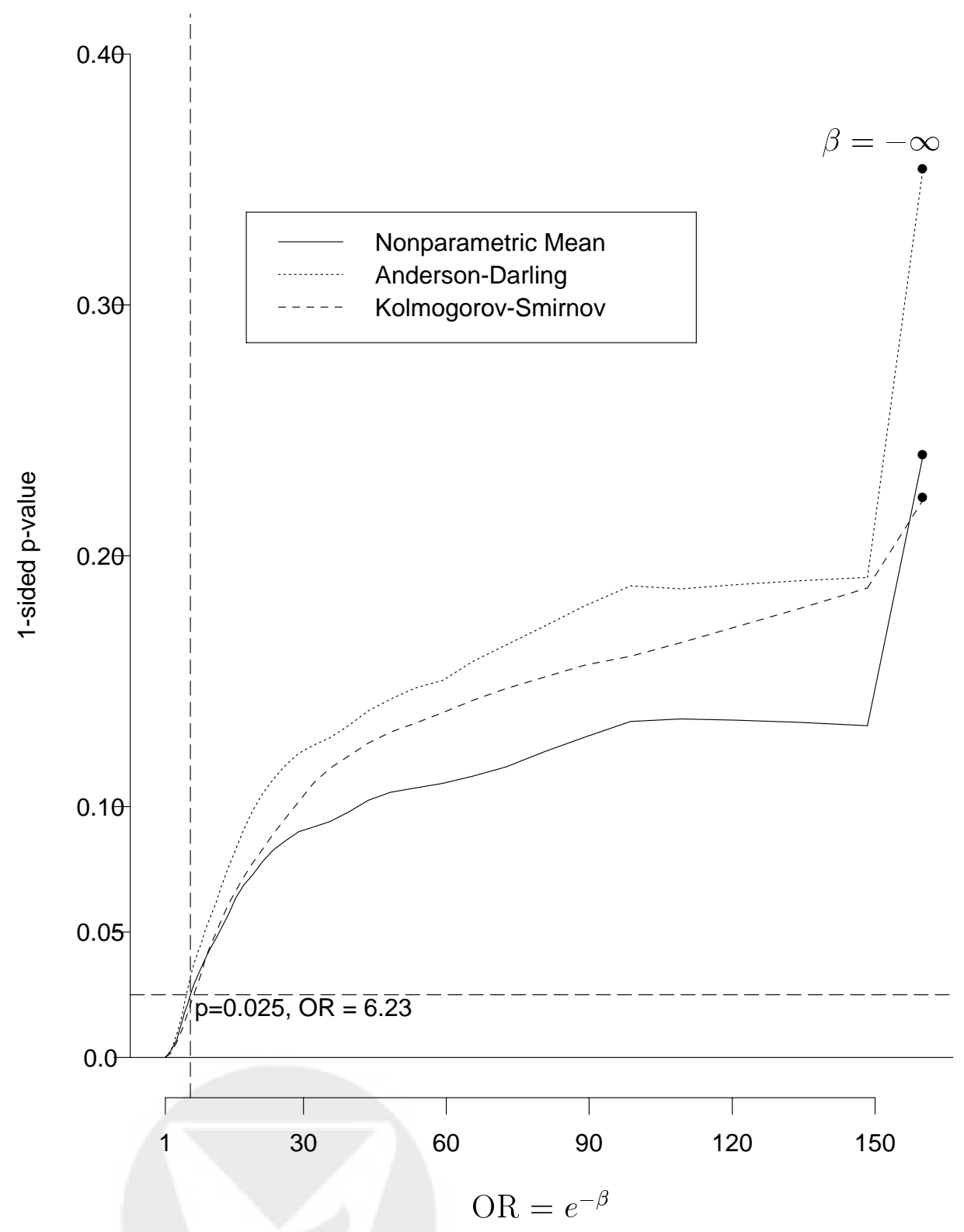

Figure 2. 


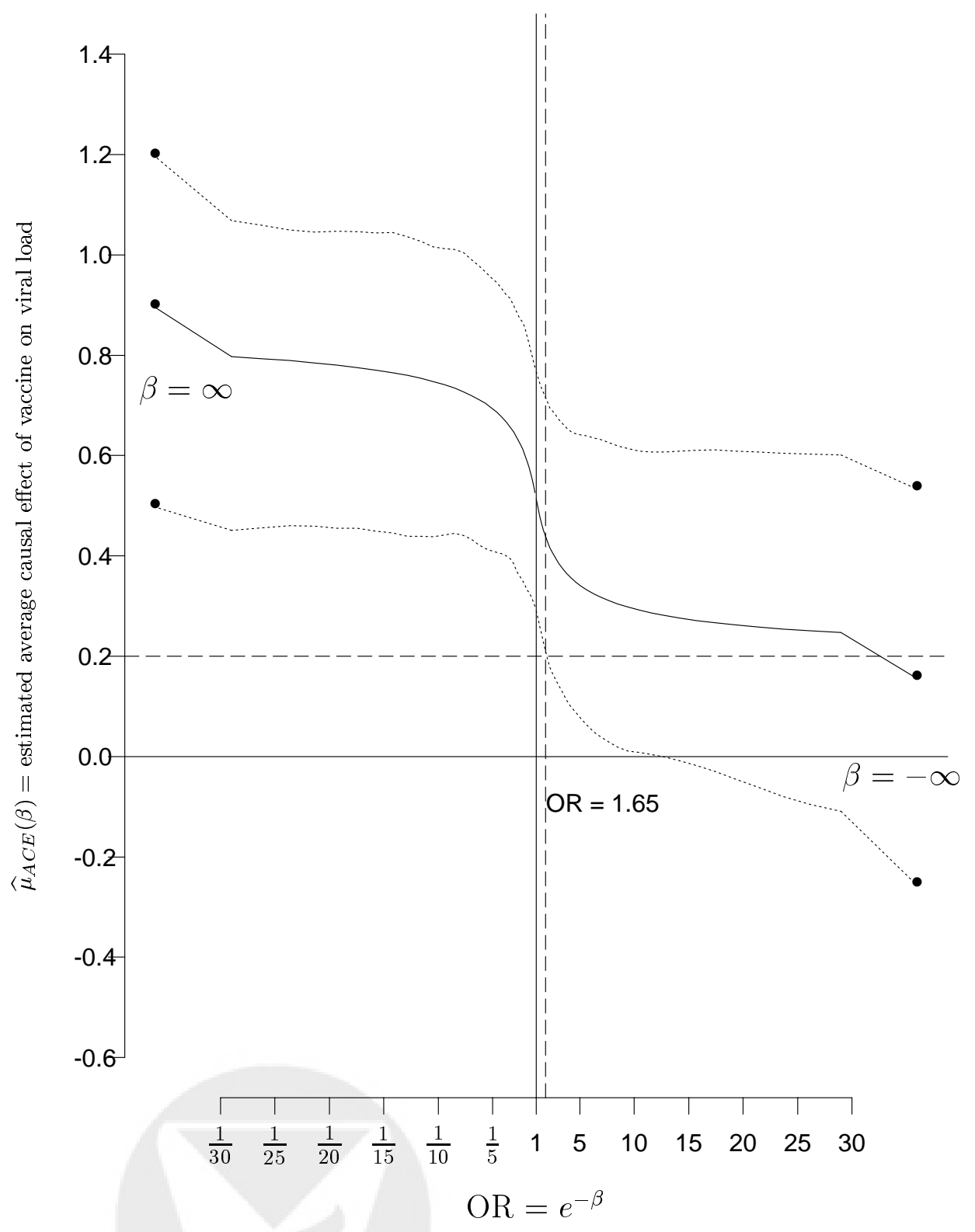

Figure 3. 